\title{
Convex Relaxation of Power Dispatch for Voltage Stability Improvement
}

\author{
Pedersen, Andreas Søndergaard; Blanke, Mogens; Jóhannsson, Hjörtur
}

Published in:

Proceedings of the 2015 IEEE Multi-Conference on Systems and Control (MSC)

Link to article, DOI:

10.1109/CCA.2015.7320827

Publication date:

2015

Document Version

Peer reviewed version

Link back to DTU Orbit

Citation (APA):

Pedersen, A. S., Blanke, M., \& Jóhannsson, H. (2015). Convex Relaxation of Power Dispatch for Voltage Stability Improvement. In Proceedings of the 2015 IEEE Multi-Conference on Systems and Control (MSC) (pp. 1528-1533). IEEE. https://doi.org/10.1109/CCA.2015.7320827

\section{General rights}

Copyright and moral rights for the publications made accessible in the public portal are retained by the authors and/or other copyright owners and it is a condition of accessing publications that users recognise and abide by the legal requirements associated with these rights.

- Users may download and print one copy of any publication from the public portal for the purpose of private study or research.

- You may not further distribute the material or use it for any profit-making activity or commercial gain

- You may freely distribute the URL identifying the publication in the public portal 


\title{
Convex Relaxation of Power Dispatch for Voltage Stability Improvement
}

\author{
Andreas S. Pedersen ${ }^{1}$, Mogens Blanke ${ }^{1,2}$ and Hjörtur Jóhannsson ${ }^{3}$
}

\begin{abstract}
A method for enhancing the voltage stability of a power system is presented in this paper. The method is based on a stability-constrained optimal power flow approach, where dispatch is done such that a maximum $L$-index is minimised for all load busses in a transmission grid. It is shown that optimal dispatch is obtainable with enhanced margins for voltage stability using a semidefinite relaxation of the optimal power flow problem, and that this problem can be formulated as semidefinite program with a quasi-convex objective. Numerical tests are performed on the IEEE-30 bus and BPA systems. The feasibility of the method is demonstrated through demonstrating that improved voltage stability margins are obtained for both systems.
\end{abstract}

\section{INTRODUCTION}

The Optimal Power Flow (OPF) is an essential tool in power system operation. It is used to obtain cost-optimal operation and to maintain the security [1] and stability [2] of a power system. The OPF is a non-convex problem, and many algorithms have been proposed to solve it. Recent efforts to solve the OPF use various convex relaxations, such as the semidefinite relaxation (SDR) [3] and second-order cone relaxation [4]. To operate a power system robustly, i.e. being able to operate with acceptable stability margins, the OPF has to be complemented by means to obtain stability margins, and incorporate these into the optimisation problem. This is referred to as stability-constrained or stability-enhancing OPF. The aim of this paper is to use the semidefinite relaxation on a stability-enhancing OPF to calculate corrective actions on a power system. Emergency operation is given particular attention. In emergencies, the power flow solution obtained as remedial action need be severely constrained to avoid further overloading. When solving a severely constrained OPF, local solvers can experience trouble finding a feasible solution. When formulated using convex relaxations, the problem has guaranteed global convergence.

Voltage instability is one of the main threats to a stable operation of modern power systems. Voltage stability refers to a power systems ability to maintain system voltages such that when the load increases, load power will increase, and such that the power and voltage are controllable [5]. Various extensions to the general OPF which incorporates voltage

\footnotetext{
*This work was supported through the SOSPO project by the Danish Council for Strategic Research under grant no. 11-116794

${ }^{1}$ Automation and Control Group at Dept. of Electrical Engineering; Technical University of Denmark, 2800 Kgs. Lyngby, Denmark \{asped, mb\}eelektro.dtu.dk

2 AMOS CoE, Institute of Technical Cybernetics, Norwegian University of Science and Technology, 7491 Trondheim, Norway

${ }^{3}$ Center for Electric Power and Energy at Dept. of Electrical Engineering; Technical University of Denmark, 2800 Kgs. Lyngby, Denmark hjjo@elektro.dtu.dk
}

stability margins - stability-constrained OPF - has been proposed in the literature, e.g. [6]. Although load power margins are the standard measure of preventive stability margins [7], other measures have been used in the literature for voltagestability-enhancing reconfiguration. Simple voltage stability indices such as the $L$-index [8] can be used as a quantitative measure for estimating the distance to stability limits. The $L$ index is a measure of distance to insolvability of the power flow equations, and the load bus with the highest $L$-index indicates the most vulnerable bus in the system. Therefore, it would be desirable to make a dispatch that minimize the maximal $L$-index on the entire system. A reason to use the $L$-index as a measure include that it can serve as an online voltage stability indicator.

Solving the OPF including constraints related to the $L$ index has been suggested in previous literature. An OPF with a maximal $L$-index constraint on each load bus was solved by [9] using an interior point method. An OPF solution with mixed $L$-index and economic objective was obtained in [10] using particle swarm optimization. A sum of squares of $L$ was minimized by [11] using a gradient approach and [12] minimised the maximal $L$-index using a genetic algorithm. The optimal reactive dispatch of renewables with regard to the $L$-index was solved in [13] using a trust region method.

The main difficulty in solving stability-constrained OPFs stem from the non-convexity of the power flow equations. As the OPF problem can be formulated as a quadratic program in the bus voltages, an SDR can be applied, which leads to convex optimization problems that can be efficiently solved, and [3] showed that their solution was exact for several benchmark systems. Various studies into which class of networks this is true is done in [3], [14]. Voltage stability is closely associated with generator reactive-power limits and these needs to be included when calculating load margins, usually through complementarity constraints [15], [16]. By including these constraints, however, the OPF problem can no longer be formulated as a quadratic program, and the SDR can no longer be employed. An approach to deal with this obstacle is to formulate, as in [17], the constrained OPF as a mixed-integer quadratic program. In this paper we account for reactive limits with detailed models of generators to better relate the reactive-power constraints to the bus voltages. By doing this, the voltage-stability measures can still be represented as quadratic indicators in the voltages, and it will be shown that SDP relaxation can be applied through introducing this technique.

The contributions of this paper include first to show how the standard semidefinite relaxation can be applied to a voltage-stability-enhancing OPF, and then, to show how this 
is possible by inclusion of detailed models in the quadratic OPF.

The paper is organized as follows: Section 2 recalls the $L$ stability index and sets up the problem formulation. A computational method for calculating the new dispatch is described in section 3. Two standard benchmark systems are presented in section 4 , and it is shown how these are optimized using a dispatch generated by the method we suggest.

\section{System Model AND Problem Formulation}

We introduce the following nomenclature: the set of busses is denoted $\mathcal{N}$, the set of load busses is $\mathcal{L} \subset \mathcal{N}, \mathcal{G} \subset \mathcal{N}$ is the set of voltage-controlled busses, and the set of transmission lines and transformers is $\mathcal{E} \subset \mathcal{N} \times \mathcal{N}$. To each bus $k$ we associate an active and reactive power injection $P_{k}^{g}, Q_{k}^{g}$ with $S_{k}^{g}=P_{k}^{g}+j Q_{k}^{g}$, an active and reactive power demand $P_{k}^{d}, Q_{k}^{d}$ with $S_{k}^{d}=P_{k}^{d}+j Q_{k}^{d}$, a complex voltage $V_{k}$ and a complex current $I_{k}$. We define vectors of bus voltages $V=\left[V_{1}, V_{2}, . ., V_{n}\right]$ and bus currents $I=\left[I_{1}, I_{2}, . ., I_{n}\right]$. The currents and voltages are related through an admittance matrix $Y$ as $I=Y V$.

The $L$-index was introduced in [8] as a simple measure of a system's voltage stability margin. A dimensionless number $0 \leq L_{k} \leq 1$ is associated to each load bus, for which 0 is no load and 1 is voltage collapse. The index is calculated as follows. The vector of bus voltages is ordered such that the first $g$ buses are those that are voltage controlled, $V=$ $\left[V_{1}, \ldots, V_{g}, V_{g+1}, \ldots, V_{n}\right]$ and $V_{g+1} . . V_{n}$ are the load busses where $n=|\mathcal{N}|$ and $g=|\mathcal{G}|$. The relationship between bus voltages and currents can be expressed by:

$$
\left[\begin{array}{c}
I_{G} \\
I_{L}
\end{array}\right]=\left[\begin{array}{ll}
Y_{G G} & Y_{G L} \\
Y_{L G} & Y_{L L}
\end{array}\right]\left[\begin{array}{l}
V_{G} \\
V_{L}
\end{array}\right]
$$

where $I_{G}, I_{L}$ and $V_{G}, V_{L}$ denote the currents and voltages at generator and load buses, respectively. By rearrangement:

$$
\left[\begin{array}{c}
V_{L} \\
I_{G}
\end{array}\right]=\left[\begin{array}{cc}
Z_{L L} & F \\
K & Y_{G G}
\end{array}\right]\left[\begin{array}{c}
I_{L} \\
V_{G}
\end{array}\right]
$$

where $F=-Y_{L L}^{-1} Y_{L G}$.

Using $F$, the $L$ index of a bus $k$ is given by

$$
L_{k}=\left|1-\sum_{i=1}^{g} F_{k i} \frac{V_{i}}{V_{k}}\right|
$$

where $F_{k i}$ is the $k, i$ element in $F$. The $L$-index of each bus represents the bus' proximity to instability, and $\max _{k \in \mathcal{L}} L_{k}$ is used as an indicator of the system's proximity to collapse.

The $L$-index assumes constant voltages at generator buses. When the reactive-power limits are activated, this will no longer be the case. An extension to the $L$-index was suggested in [18] to include these effects. This approach will be applied in the stability-constrained OPF. The network is appended with the internal node of the machines. The electrical equations for generators can be written:

$$
\begin{aligned}
& e_{d}^{\prime \prime}=v_{d}+r_{a} i_{d}-\left(x_{q}^{\prime \prime}-x_{l}\right) i_{q} \\
& e_{q}^{\prime \prime}=v_{q}+r_{a} i_{q}+\left(x_{d}^{\prime \prime}-x_{l}\right) i_{d}
\end{aligned}
$$

Under the assumption $x_{d}^{\prime \prime} \approx x_{q}^{\prime \prime}$, the internal voltage are calculated from behind a constant impedance $Z_{k}=R_{a}+$ $j\left(X_{d}^{\prime \prime}-X_{l}\right)$ as:

$$
E_{k}=V_{k}+Z_{k} I_{k}
$$

The admittance matrix is extended to include the internal nodes. Let $Y_{g g}=\operatorname{diag} Z_{k}$

$$
\left[\begin{array}{ccc}
Y_{g g} & -Y_{g g} & 0 \\
-Y_{g g} & Y_{G G}+Y_{g g} & Y_{G L} \\
0 & Y_{L G} & Y_{L L}
\end{array}\right]\left[\begin{array}{c}
E_{G} \\
V_{G} \\
V_{L}
\end{array}\right]=\left[\begin{array}{c}
I_{G} \\
0 \\
I_{L}
\end{array}\right]
$$

By Kron reduction, the new $L$-index can be calculated using [18]:

$$
F^{\prime}=-Z_{L L}^{\prime} Y_{L G}^{\prime}
$$

where $Z_{L L}^{\prime}=\left(Y_{L L}-Y_{L G}\left(Y_{G G}+Y_{g g}\right)^{-1} Y_{G L}\right)^{-1}$ and $Y_{L G}^{\prime}=$ $Y_{L G}\left(Y_{G G}+Y_{g g}\right)^{-1} Y_{g g}$. The extended $L$-index for a load bus $k$ is then calculated using

$$
L_{k}^{\prime}=\left|1-\sum_{i=1}^{g} F_{k i}^{\prime} \frac{E_{i}}{V_{k}}\right|
$$

Protective controls are present in synchronous machines in order to avoid overheating in the field windings. When the protective controls are active, they limit the machines reactive power output and instantaneously change the voltage-control capabilities of the machine (see [19] for a study of the effects on voltage stability). The reactive limits of a machine has origin in the limitations on the currents in field and armature windings [20].

For $X_{d} \approx X_{q}$ the field excitation voltage is determined by [20]:

$$
\left|E_{f, k}\right|=\left|X_{a d} i_{f d}\right|=\left|V_{k}+\left(R_{a}+j X_{q}\right) I_{k}\right|
$$

The field voltage and armature current will be constrained by $E_{f, k}^{\max }$ and $I_{k}^{\max }$.

The voltage-stability measure and the machine limitations discussed above will be included to find a stability enhanced power dispatch. The stability enhancing OPF then takes the form,

$$
\begin{aligned}
\min & \max _{k \in \mathcal{L}} L_{k} \\
\text { s.t. } & I_{k}^{*} V_{k}=S_{k}^{g}-S_{k}^{d}, \quad \forall k \in \mathcal{N} \\
& P_{k}^{\min } \leq P_{k} \leq P_{k}^{\max }, \quad \forall k \in \mathcal{N} \\
& Q_{k}^{\min } \leq Q_{k} \leq Q_{k}^{\max }, \quad \forall k \in \mathcal{N} \\
& V_{k}^{\min } \leq\left|V_{k}\right| \leq V^{\max }, \quad \forall k \in \mathcal{N} \\
& \left|S_{l, m}\right| \leq S_{l, m}^{\max }, \quad \forall(l, m) \in \mathcal{E} \\
& \left|E_{f, k}\right| \leq E_{f, k}^{\max }, \quad \forall k \in \mathcal{G} \\
& \left|I_{k}\right| \leq I_{k}^{\max }, \quad \forall k \in \mathcal{G}
\end{aligned}
$$

The constraint (11b) is the nodal power balance, constraint (11c) the real power generation limit, constraint (11e) the bus voltage magnitude limit, constraint (11f) the transmission line flow limit, constraint (11g) the field voltage limit and constraint (11h) is the armature current limit. 
The optimization (11) defines the voltage-enhancing OPF. The non-convexity of (11) stems from the nodal balance (11b), which will be convexified in the next section using the SDR.

\section{Dispatch COMPUTATION}

The stability-enhancing OPF (11) is now reformulated such that it fits into a framework of standard semidefinite relaxation [3]. This is done as follows.

Let $e_{k}$ denote the standard basis vector in $\mathbb{R}^{n}$. The optimization will be done with the internal nodes. To this end, define the vector $E=\left[E_{1}, \ldots, E_{g}, V_{g+1}, \ldots, V_{n}\right]$. To relate the bus voltages $V$ to elements in the $E$ vector, define a matrix $M=\left(\mathbf{1}+\operatorname{diag}(Z \mathbf{0}) Y_{e}\right)^{-1}$, such that $V=M E$.

Note that the left-hand side of (11b) can be expressed as $I_{k}^{*} V_{k}=V^{H} M^{H} Y^{H} e_{k} e_{k}^{T} M V$, which in the sequel is used to eliminate $I_{k}$. Define the Hermitian matrices

$$
\begin{aligned}
& T_{P, k}=\frac{1}{2}\left(M^{H} Y^{H} e_{k} e_{k}^{T} M+M^{H} e_{k} e_{k}^{T} Y M\right) \\
& T_{Q, k}=-\frac{j}{2}\left(M^{H} Y^{H} e_{k} e_{k}^{T} M-M^{H} e_{k} e_{k}^{T} Y M\right)
\end{aligned}
$$

Using the fact that $E^{H} T_{P, k} E=\operatorname{tr} T_{P, k} E E^{H}$, and introducing $W=E E^{H}$, the nodal power balance (11b) and generation constraints (11c)-(11d) can be reformulated as

$$
\begin{aligned}
P_{k}^{\min } & \leq \operatorname{tr} T_{P, k} F+P_{k}^{d} \leq P_{k}^{\max } \\
Q_{k}^{\min } & \leq \operatorname{tr} T_{Q, k} F+Q_{k}^{d} \leq Q_{k}^{\max }
\end{aligned}
$$

For all load busses, $P_{k}^{\min }, P_{k}^{\max }, Q_{k}^{\min }, Q_{k}^{\max }$ will be set to zero.

The transmission line limits (11f) are reformulated using a Schur complement with matrices $T_{L P, l m}$ and $T_{L Q, l m}$ as defined in [21]:

$$
\left[\begin{array}{ccc}
S_{l, m}^{\max } & -\operatorname{tr} T_{L P, l m} H & -\operatorname{tr} T_{L Q, l m} W \\
-\operatorname{tr} T_{L P, l m} H & 1 & 0 \\
-\operatorname{tr} T_{L Q, l m} H & 0 & 1
\end{array}\right] \succeq 0
$$

The squared voltage magnitudes are in the diagonal of $W$, and the bus magnitude limits (11e) can hence be written:

$$
V_{k}^{\min ^{2}} \leq\left(M W M^{H}\right)_{k k} \leq V_{k}^{\max 2} .
$$

The field voltage limits are rewritten by squaring (10):

$$
\begin{aligned}
\left|E_{f, k}\right|^{2} & \\
& =\left(V_{k}+j X_{q} I_{k}+R_{a} I_{k}\right)^{*}\left(V_{k}+j X_{q} I_{k}+R_{a} I_{k}\right) \\
& =\operatorname{tr} G_{k} W
\end{aligned}
$$

where the matrix $G_{k}=e_{k}^{T}\left(1+\left(j X_{q}+R_{a}\right) Y\right) M M^{H}((1+$ $\left.\left(j X_{q}+R_{a}\right)^{*} Y^{H}\right) e_{k}$.

Squaring the $L$-index Eq.(9), it can be expressed by $W$ as:

$$
\begin{gathered}
L_{k}^{\prime 2}=\frac{1}{W_{k k}}\left(W_{k k}-\sum_{i \in \mathcal{G}} F_{k i}^{\prime} W_{i k}-\sum_{i \in \mathcal{G}} F_{k i}^{\prime *} W_{k i}\right. \\
\left.+\sum_{i \in \mathcal{G}} \sum_{j \in \mathcal{G}} F_{k i}^{\prime} F_{k j}^{*} W_{i j}\right)
\end{gathered}
$$

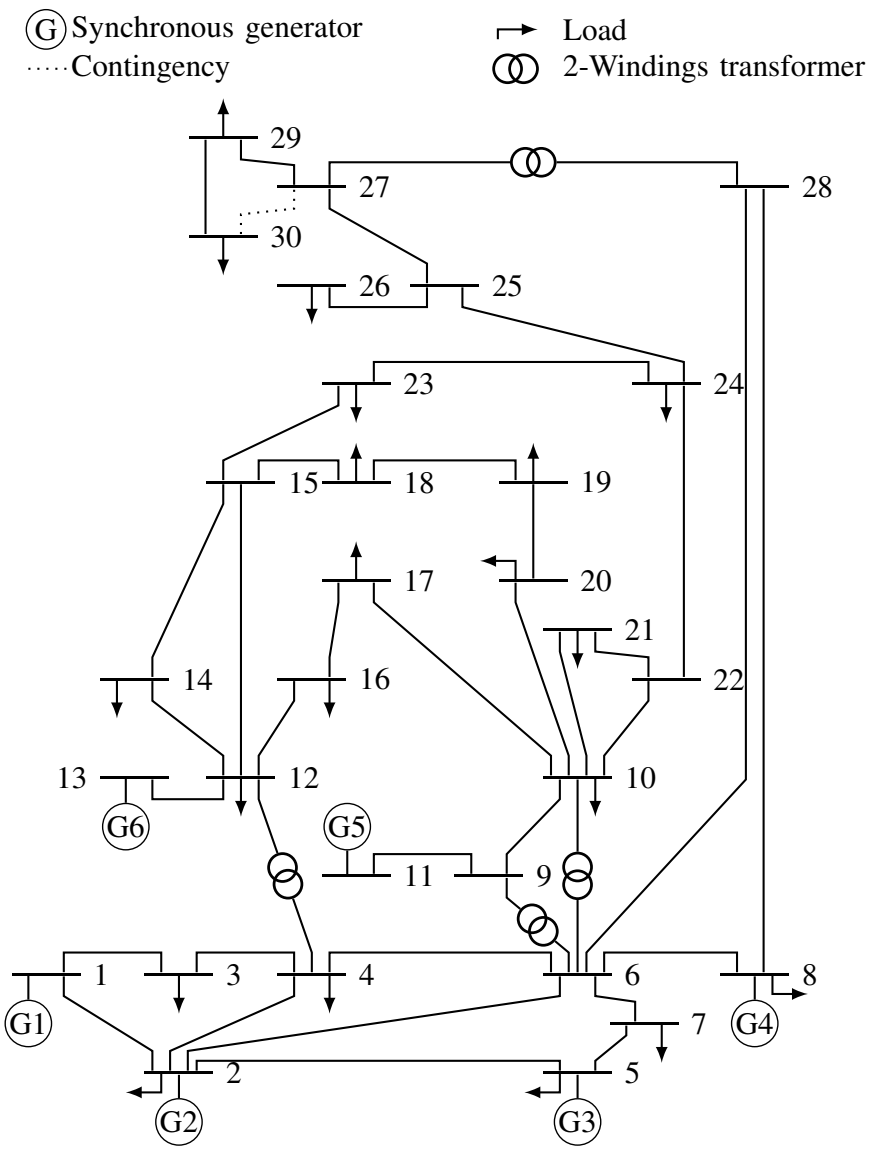

Fig. 1. IEEE-30 bus benchmark system, where the dotted line shows the considered contingency.

The variables $y_{k}=L_{k}^{\prime 2} W_{k k}$ is introduced. Using $y_{k}$, the stability-enhancing OPF, consisting of (14), (15), (17), (16), (18) and (19), results in:

$$
\begin{aligned}
& \min \max _{k \in \mathcal{L}}\left\{\frac{y_{k}}{W_{k k}}\right\} \\
& -y_{k}-\sum_{i \in \mathcal{G}} F_{k i}^{\prime} W_{i k}-\sum_{i \in \mathcal{G}} F_{k i}^{*} W_{k i} \\
& +\sum_{i \in \mathcal{G}} \sum_{j \in \mathcal{G}} F_{k i}^{\prime} F_{k j}^{\prime *} W_{i j} \leq 0, \quad \forall k \in \mathcal{L} \\
& P_{k}^{\min } \leq \operatorname{tr} T_{P, k} W+P_{k}^{d} \leq P_{k}^{\max }, \quad \forall k \in \mathcal{N} \\
& Q_{k}^{\min } \leq \operatorname{tr} T_{Q, k} W+Q_{k}^{d} \leq Q_{k}^{\max }, \quad \forall k \in \mathcal{L} \\
& V_{k}^{\min ^{2}} \leq\left(M W M^{H}\right)_{k k} \leq V_{k}^{\max 2}, \quad \forall k \in \mathcal{N} \\
& \operatorname{tr} G_{k} W \leq E_{f, k}^{\max 2}, \quad \forall k \in \mathcal{G} \\
& \left(M^{H} U^{H} W U M\right)_{k k} \leq I_{k}^{\max 2}, \quad \forall k \in \mathcal{G} \\
& {\left[\begin{array}{ccc}
S_{l, m}^{\max } & -\operatorname{tr} T_{L P, l m} W & -\operatorname{tr} T_{L Q, l m} W \\
-\operatorname{tr} T_{L P, l m} W & 1 & 0 \\
-\operatorname{tr} T_{L Q, l m} W & 0 & 1
\end{array}\right]} \\
& \succeq 0, \quad \forall(l, m) \in \mathcal{E} \\
& W=E E^{H}
\end{aligned}
$$

The semidefinite relaxation is now done by replacing $(20 \mathrm{j})$ 


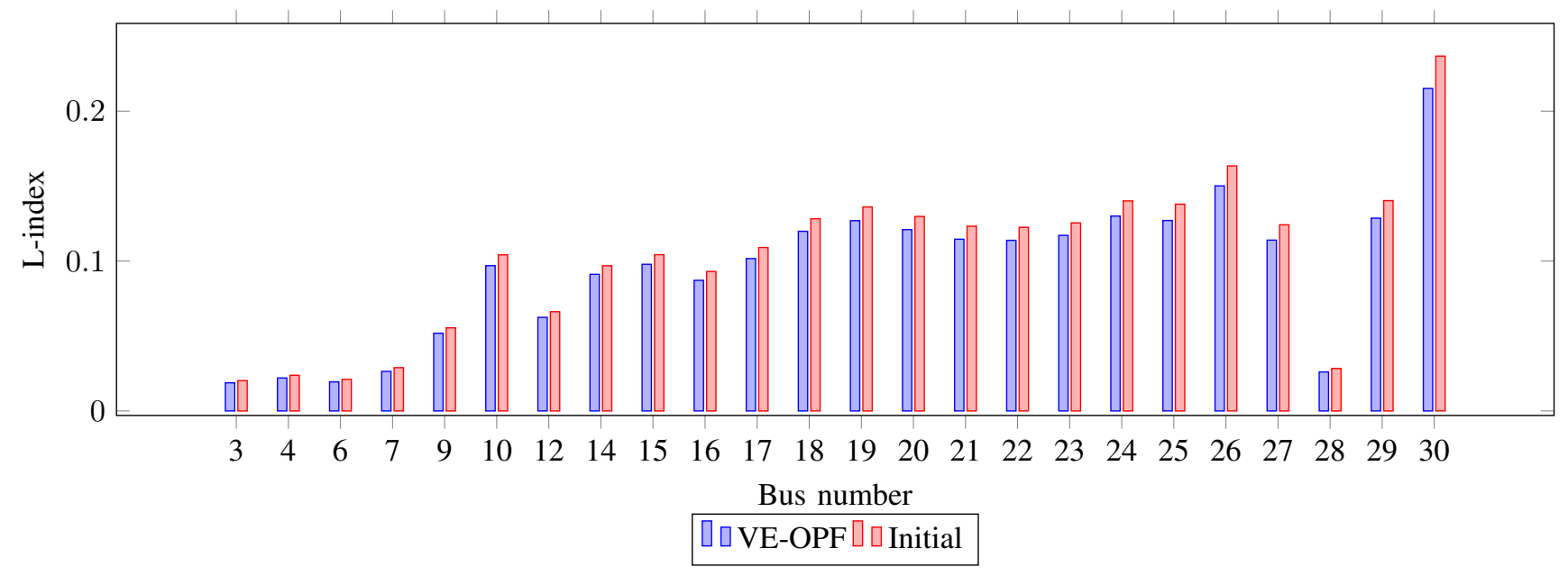

Fig. 2. $L$-index of all load busses on IEEE 30-bus benchmark system. A lower index indicates a better voltage stability margin.

with $W \succeq 0$. The resulting program is quasi-convex, and its solution $W^{*}$ gives a lower bound on the optimal value of (11). If the solution has rank-1, the solution is exact and can be obtained from $W^{*}=E E^{H}$.

A bisection method can now be used to solve the quasiconvex optimization. This is done by finding a convex function $\theta_{t}\left(W, y_{k}\right)$ for which the objective functions $t$-sublevel set is the 0 -sublevel set of $\theta_{t}$. For a given $t$ we solve the feasibility problem:

$$
\begin{aligned}
\text { find } & W \\
\text { s.t. } & (20 b)-(20 i) \\
& W \succeq 0 \\
& \theta_{t}\left(W, y_{k}\right) \leq 0, \quad \forall k \in \mathcal{L}
\end{aligned}
$$

Using bisection to maximize $t$, the problem (21) can be solved by a series of SDPs. This problem is equivalent to solving:

$$
\begin{aligned}
\max & t \\
\text { s.t. } & -t W_{k k}+y_{k} \geq 0, \quad \forall k \in \mathcal{L} \\
& (20 b)-(20 i) \\
& W \succeq 0
\end{aligned}
$$

The optimization (22) is a main result of this paper. It shows how a semidefinite relaxation is obtained from the original non-convex voltage-enhancing OPF (11) such that the optimization (22) is solvable using standard SDP solvers.

The next section will demonstrate the voltage-stabilityenhancing OPF in two case studies and show how enhanced voltage stability is obtained with the proposed method.

\section{EXAMPLE}

The method is tested on two benchmark systems. The first test is done on the IEEE-30 bus system, and shows the voltage magnitude improvements using the voltage-stability enhancing OPF (VE-OPF) following a contingency. The second test is a dynamic simulation, where the method is applied as a way of calculating a remedial action for a system in an emergency state.

\section{A. IEEE 30-bus system}

The IEEE 30-bus system (Figure 1) consists of six generator busses and 24 load busses. The standard IEEE 30-bus system [22] is modified to increase the loading on the system. This was done by increasing all loads by a factor of 1.25.

To illustrate the effects of improving the $L$-index, a contingency is considered where the line 27-30 is disconnected. Two different dispatches are considered for the precontingency system, the default dispatch and the voltagestability enhanced OPF dispatch. The latter was found from (20) with a bisection precision of $\epsilon=1 e-4$. The optimization terminated with a rank $=1$ solution $W^{*}$, which allowed recovery of the exact solution. The resulting $L$-index of all load busses is shown in Figure 2.

Using these dispatches, the line 27-30 disconnection contingency is applied and the resulting bus voltages are examined. The $L$-index and voltage magnitude of bus 30 is shown in Table I.

\begin{tabular}{rrr}
\hline & \multicolumn{2}{c}{ Post contingency } \\
\cline { 2 - 3 } & Initial & VE-OPF \\
\hline$L_{30}$ & 0.3310 & 0.2966 \\
$\left|V_{30}\right|$ & $0.89 \mathrm{pu}$ & $0.95 \mathrm{pu}$ \\
\hline
\end{tabular}

TABLE I

RESULT OF BUS VOLTAGE MAGNITUDE UNDER CONTINGENCY FOR IEEE 30-BUS SYSTEM.

From the results in Table $\mathrm{I}$ it is clear that the voltagestability enhanced dispatch has a better robustness against disturbances in the grid. For the default dispatch, the voltage on bus 30 drops to $0.89 \mathrm{pu}$, where an improvement is shown in the voltage-profile using the dispatch from (20), where voltage only drops to $0.95 \mathrm{pu}$. 

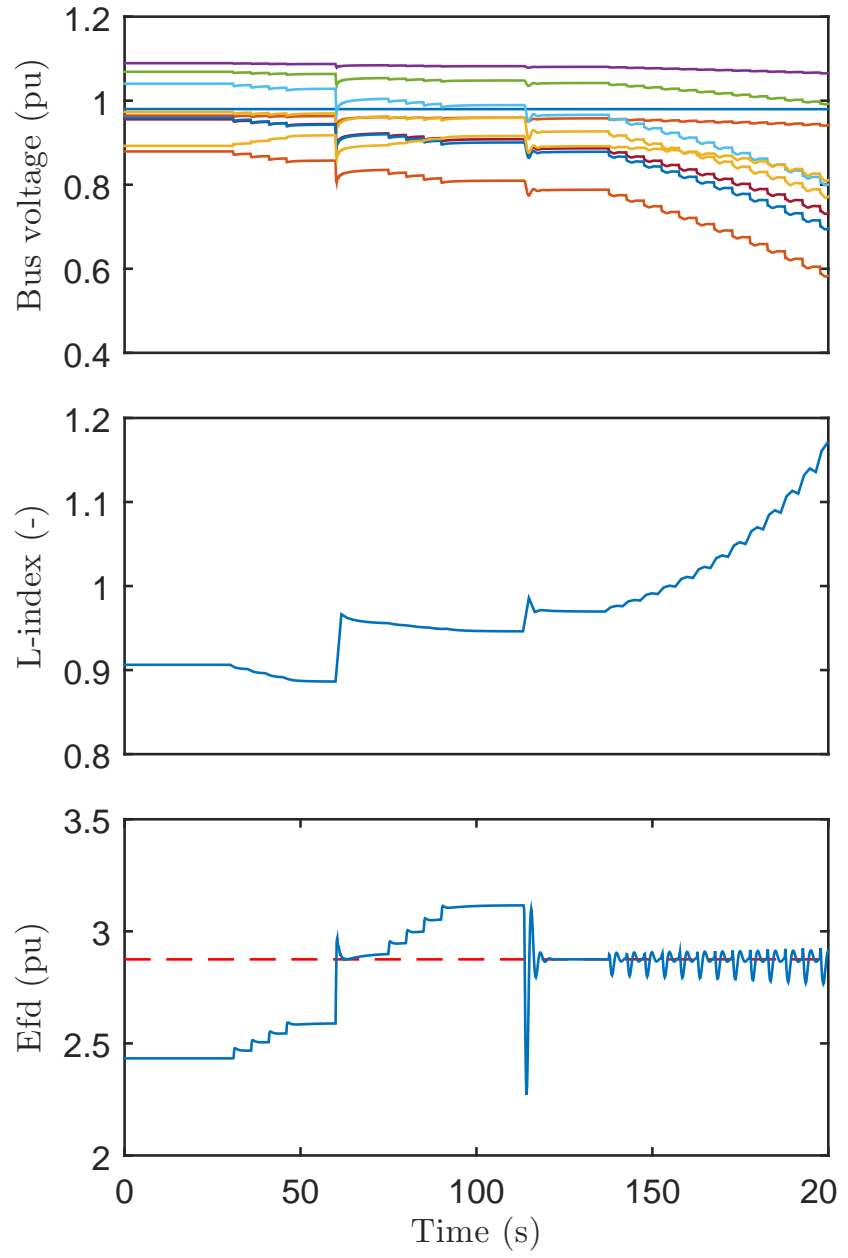

Fig. 3. Time simulation of BPA system, with a fault injected at time $t=60$ s.

\section{B. BPA dynamic case study}

The second test case is a dynamic simulation where the proposed method is employed to calculate a remedial action for a system in an emergency state. The test system from [23] is employed to show feasibility of the method. The system (cf. Figure 5) is a 11-bus, 3 generator system, with a generating area on the left consisting of generators $G 1$ and $G 2$, which are connected to a local area on the right. Generator $G 1$ represents an infinite bus. Generators $G 2$ and $G 3$ are voltage controlled. Generator $G 3$ is equipped with an over-excitation limiter (OXL). The others never reach excitation limits in the test case. An On-Load Tap Changer (OLTC) is connected to bus 11 to maintain the load side voltage.

The system is operated close to its stability limits. After one of the lines between bus 6 and 7 is tripped, the OLTC at bus 11 will try to maintain the load voltage, but the operation point ends up beyond the point of maximal power deliverable and the result is a voltage collapse. A time domain simulation of the system is shown in Figure 3.

The bus voltage at bus 11 is initially too low, which the OLTC recovers. At time $t=60 \mathrm{~s}$, one of the lines between
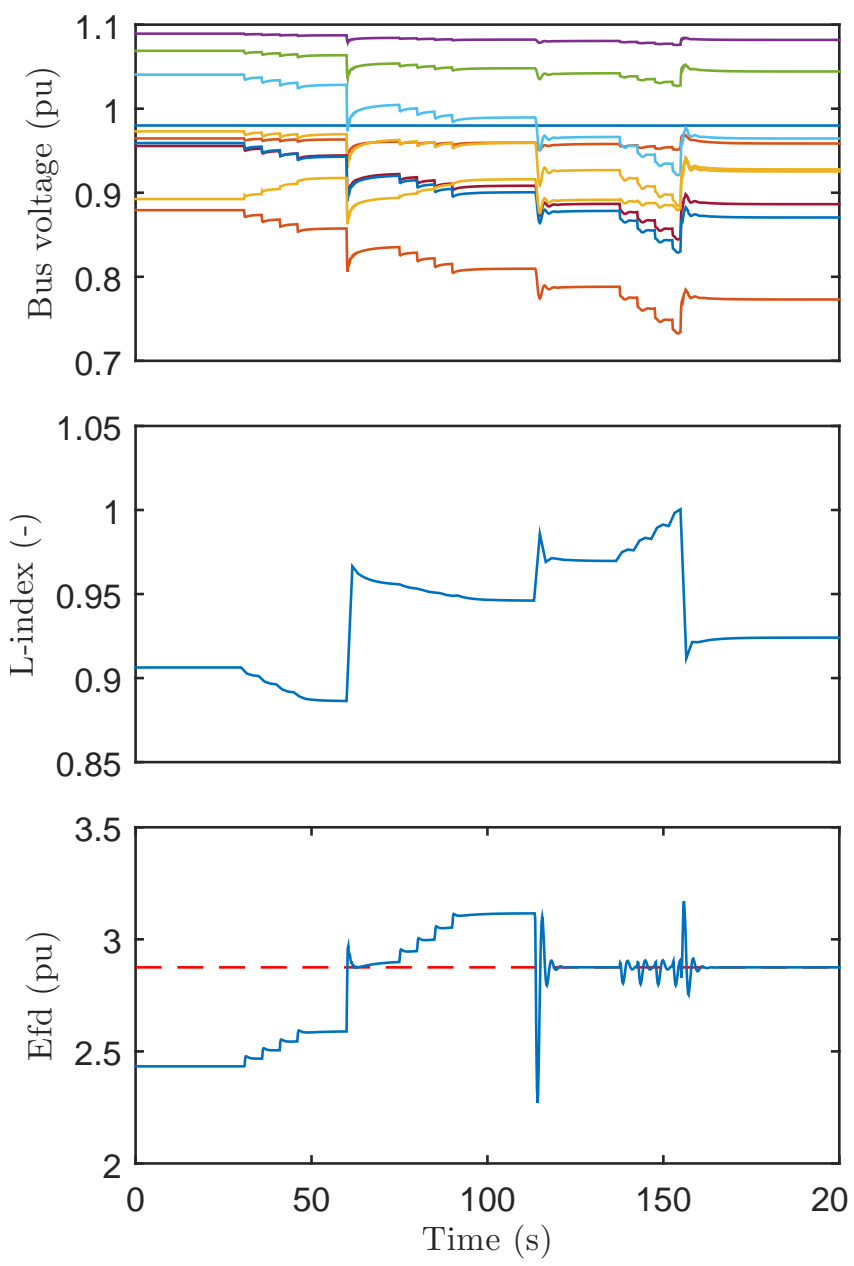

Fig. 4. Time simulation of BPA system, with a fault injected at time $t=60$ s. The $L$-index is used as an emergency indicator, and when $L_{11}>1$ at $t=155 \mathrm{~s}$ a remedial action is applied.

bus 6 and 7 is tripped. When this happens, the field voltage of generator $G 3$ crosses its maximal limit. The OXL is allowed to operate at a higher field voltage for a limited period of time. Within this period, the OLTC successfully recovers the voltage at bus 11 , but at time $t=110 \mathrm{~s}$ the OXL is activated and at time $t=155 \mathrm{~s}$ the $L$-index indicates that the system is voltage unstable. The OLTC continuously tries to maintain the load side voltage at bus 11 , but a voltage collapse is the result.

Remidial action: We will now use the SDP formulation to calculate the necessary load-shedding to avoid a voltage collapse. In this case we replace the voltage-enhancing objective with an objective to minimize the load shedding at bus 11 , and a constraint relating to the $L$-index is added, constraining $L_{k}^{\prime}<0.95$ for all load busses.

The optimization obtains a rank-one solution $W^{*}$ to (22), such that the solution is exact. The remedial action takes place from time $t=155 \mathrm{~s}$, by the shedding of $391 \mathrm{MW}$ load on bus 11. By this remedial action, the system is able to recover stability, as seen from the $L$-index. The resulting response is shown in Figure 4. 


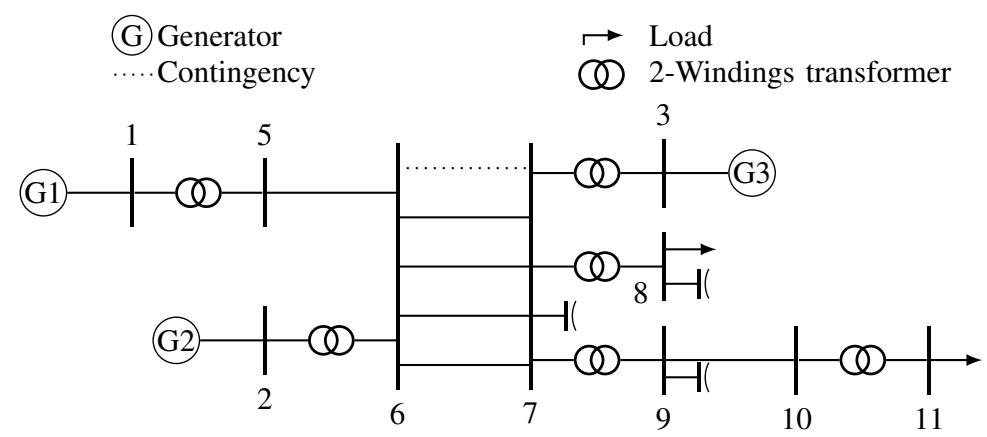

Fig. 5. BPA test system used for dynamic simulation. The dotted line indicates the considered contingency.

\section{CONCLUSION}

This paper presented a method for solving a stabilityenhancing optimal power flow problem using a semidefinite relaxation, resulting in a quasi-convex semidefinite program. The method was tested on two benchmark systems, which showed feasibility of the method to improve the voltagestability of a power system.

\section{REFERENCES}

[1] F. Capitanescu, J. Martinez Ramos, P. Panciatici, D. Kirschen, A. Marano Marcolini, L. Platbrood, and L. Wehenkel, "State-of-theart, challenges, and future trends in security constrained optimal power flow," Electric Power Systems Research, vol. 81, no. 8, pp. 1731-1741, Aug. 2011.

[2] S. Granville, J. Mello, and a.C.G. Melo, "Application of interior point methods to power flow unsolvability," IEEE Transactions on Power Systems, vol. 11, no. 2, pp. 1096-1103, 1996.

[3] J. Lavaei and S. H. Low, "Zero Duality Gap in Optimal Power Flow Problem," IEEE Transactions on Power Systems, vol. 27, no. 1, pp. 92-107, Feb. 2012.

[4] R. a. Jabr, "A conic quadratic format for the load flow equations of meshed networks," IEEE Transactions on Power Systems, vol. 22, no. 4, pp. 2285-2286, 2007.

[5] C. Concordia, "Voltage instability," International Journal of Electrical Power \& Energy Systems, vol. 13, no. 1, pp. 14-20, 1991.

[6] W. Rosehart, C. Canizares, and V. Quintana, "Optimal power flow incorporating voltage collapse constraints," 1999 IEEE Power Engineering Society Summer Meeting. Conference Proceedings, 1999.

[7] T. van Cutsem and C. Vournas, Voltage Stability of Electric Power Systems, ser. Power Electronics and Power Systems. Springer US, 2007.

[8] P. Kessel and H. Glavitsch, "Estimating the voltage stability of a power system," Power Delivery, IEEE Transactions on, no. 3, pp. 346-354, 1986

[9] S. Kim, T.-Y. Song, M.-H. Jeong, B. Lee, Y.-H. Moon, J.-Y. Namkung, and G. Jang, "Development of Voltage Stability Constrained Optimal Power Flow ( VSCOPF )," Power Engineering Society Summer Meeting, 2001, vol. 3, no. C, pp. 1664-1669, 2001.

[10] M. Abido, "Optimal power flow using particle swarm optimization," International Journal of Electrical Power \& Energy Systems, vol. 24 no. 7, pp. 563-571, 2002.
[11] D. Thukaram, L. Jenkins, and K. Visakha, "Optimum allocation of reactive power for voltage stability improvement in ACDC power systems," p. 237, 2006.

[12] D. Devaraj and J. P. Roselyn, "Genetic algorithm based reactive power dispatch for voltage stability improvement," International Journal of Electrical Power \& Energy Systems, vol. 32, no. 10, pp. 1151-1156, 2010.

[13] V. S. Sravan Kumar, K. Krishna Reddy, and D. Thukaram, "Coordination of Reactive Power in Grid-Connected Wind Farms for Voltage Stability Enhancement," pp. 2381-2390, 2014.

[14] B. Zhang and D. Tse, "Geometry of feasible injection region of power networks," 2011 49th Annual Allerton Conference on Communication, Control, and Computing, Allerton 2011, pp. 1508-1515, 2011.

[15] C. D. Vournas, M. Karystianos, and N. G. Maratos, "Bifurcation points and loadability limits as solutions of constrained optimization problems," Power Engineering Society Summer Meeting, 2000, vol. 00 , no. c, pp. $1883-1888,2000$.

[16] W. Rosehart, C. Roman, and A. Schellenberg, "Optimal power flow with complementarity constraints," IEEE Transactions on Power Systems, vol. 20, no. 2, pp. 813-822, 2005.

[17] D. K. Molzahn, V. Dawar, B. C. Lesieutre, and C. L. DeMarco, "Sufficient conditions for power flow insolvability considering reactive power limited generators with applications to voltage stability margins," 2013 IREP Symposium Bulk Power System Dynamics and Control - IX Optimization, Security and Control of the Emerging Power Grid, pp. 1-11, Aug. 2013.

[18] Y. Wang, C. Wang, F. Lin, W. Li, L. Y. Wang, and J. Zhao, "Incorporating Generator Equivalent Model Into Voltage Stability Analysis," IEEE Transactions on Power Systems, vol. 28, no. 4, pp. 4857-4866, Nov. 2013.

[19] C. Vournas, G. Manos, P. Sauer, and M. Pai, "Effect of overexcitation limiters on power system long-term modeling," IEEE Transactions on Energy Conversion, vol. 14, no. 4, pp. 1529-1536, 1999.

[20] P. Kundur, Power System Stability and Control. McGraw-Hill, 1994

[21] D. K. Molzahn, "Application of Semidefinite Optimization Techniques to Problems in Electric Power Systems," 2013.

[22] University of Washington, "Power system test case archive," http://www.ee.washington.edu/research/pstca/.

[23] G. K. Morison, B. Gao, and P. Kundur, "Voltage stability analysis using static and dynamic approaches," Power Systems, IEEE Transactions on, vol. 8, no. 3, pp. 1159-1171, 1993. 